\title{
Multi-dimensional models of Type la supernovae
}

\author{
F. K. Röpke ${ }^{* 1,2}$, M. Fink ${ }^{2}$, W. Hillebrandt ${ }^{2}$, M. Kromer ${ }^{2}$, R. Pakmor ${ }^{3}$, I. R. Seitenzahl ${ }^{2}$, \\ and S. A. Sim ${ }^{4}$ \\ ${ }^{1}$ Institut für Theoretische Physik und Astrophysik, Universität Würzburg, Am Hubland, \\ D-97074 Würzburg, Germany \\ ${ }^{2}$ Max-Planck-Institut für Astrophysik, Karl-Schwarzschild-Str. 1, D-85748 Garching, Germany \\ ${ }^{3}$ Heidelberg Institute for Theoretical Studies Schloss-Wolfsbrunnenweg 35, \\ D-69118 Heidelberg, Germany \\ ${ }^{4}$ Research School of Astronomy and Astrophysics, Mount Stromlo Observatory, Cotter Road, \\ Weston Creek, ACT 2611, Australia \\ E-mail: fritzempa-garching.mpg.de
}

Type Ia supernovae (SNe Ia) exhibit a significant diversity in their observables suggesting that a variety of progenitor channels and/or explosion mechanisms contribute to this class of objects. Understanding the origin of the diversity is essential for applying SNe Ia as distance indicators in cosmology and for studying their role in cosmic nucleosynthesis.

Multi-dimensional simulations of SN Ia explosions allow for a consistent treatment of the underlying physical mechanisms. Consequently, their predictive power enables a direct comparison with observations. This way, the validity of different explosion scenarios can be assessed.

Based on a comprehensive sequence of modeling taking into account aspects of population synthesis, multi-dimensional hydrodynamic explosion simulations, nucleosynthetic postprocessing, and radiative transfer calculations, the capability of different explosion scenarios to reproduce SN Ia observations is discussed. Although traditional Chandrasekhar-mass models are promising candidates for explaining "normal" SNe Ia, the corresponding progenitors may not be numerous enough to account for their rate. Explosions of sub-Chandrasekhar mass white dwarfs provide a potential alternative. The observables predicted for violent mergers of two white dwarfs, however, resemble a class of sub-luminous SNe Ia rather than the bulk of normal events.

An accurate modeling of the nucleosynthesis in SN Ia explosions is found to be critical not only for drawing conclusions on their impact on galactic chemical evolution, but also for the predicted observables.

11th Symposium on Nuclei in the Cosmos

19-23 July 2010

Heidelberg, Germany.

\footnotetext{
*Speaker.
} 


\section{Introduction}

The most fundamental problem in establishing a theoretical picture of Type Ia supernova (SN Ia) explosions is the lack of knowledge of the progenitor system. Therefore the initial conditions of the explosion phase remain uncertain. Although the association of SNe Ia with thermonuclear explosions of carbon-oxygen white dwarf (WD) stars in binary systems remains undisputed, different evolutionary paths have been suggested [1]. Following the last hours before the onset of the explosions in numerical simulations is a tremendous challenge and the details of binary evolution in many of the suggested scenarios is out of reach for multi-dimensional hydrodynamical modeling.

While modeling the explosion process itself remains a demanding task, there has been substantial progress in this field over the last decade. Multi-dimensional hydrodynamical simulations have been carried out and from these synthetic observables have been derived by means of radiative transfer simulations. This allows us to model the various scenarios that have been proposed to explain SNe Ia and predict their observable properties. Population synthesis studies provide a complementary approach of testing SN Ia scenarios by estimating the relative frequencies of different explosion scenarios. Thus, although a straightforward multi-dimensional modeling of SN Ia explosions from the binary evolution of the progenitor system to the formation of observables remains out of reach in the foreseeable future, the currently available tools and approaches do provide means of constraining SN Ia scenarios.

\section{Modeling sequence}

To test SN Ia scenarios, we have performed various sequences of numerical simulations. Initial progenitor system parameters can be motivated by population synthesis studies that help to identify which systems might be abundant enough to explain the observed rates [2]. In the case of WD-WD mergers, the pre-explosion binary evolution is simulated [3]; in all other cases the explosion is triggered by hand in an assumed progenitor configuration. After ignition, the thermonuclear explosion is followed in multi-dimensional hydrodynamic simulations. Since full reaction networks are computationally too expensive to run concurrently with the hydrodynamics simulation, the burning is modeled in a coarse approximation including only five species [4]. This is sufficient to estimate the nuclear energy release that drives the dynamics of the explosion. To determine the details of the nucleosynthesis, however, an additional step needs to be taken. This step is based on tracer particles that are placed in the exploding object and advected along with the explosion (see the contribution of I. Seitenzahl). The tracer particles record thermodynamic trajectories. These are then used as an input for a large reaction network that is solved to determine the nucleosynthesis in the explosion [5, 6, 7]. By mapping the results into radiative transfer calculations and deriving synthetic observables (light curves and spectra) [8,9], a direct comparison with observations is possible. This connects the end of the modeling pipeline to astronomical data, making it possible to answer the question of whether the event has properties consistent with the $\mathrm{SNe}$ Ia we observe.

\section{Violent WD-WD mergers}

A scenario for $\mathrm{SNe}$ Ia that is favored in some population synthesis calculations [2] is the 

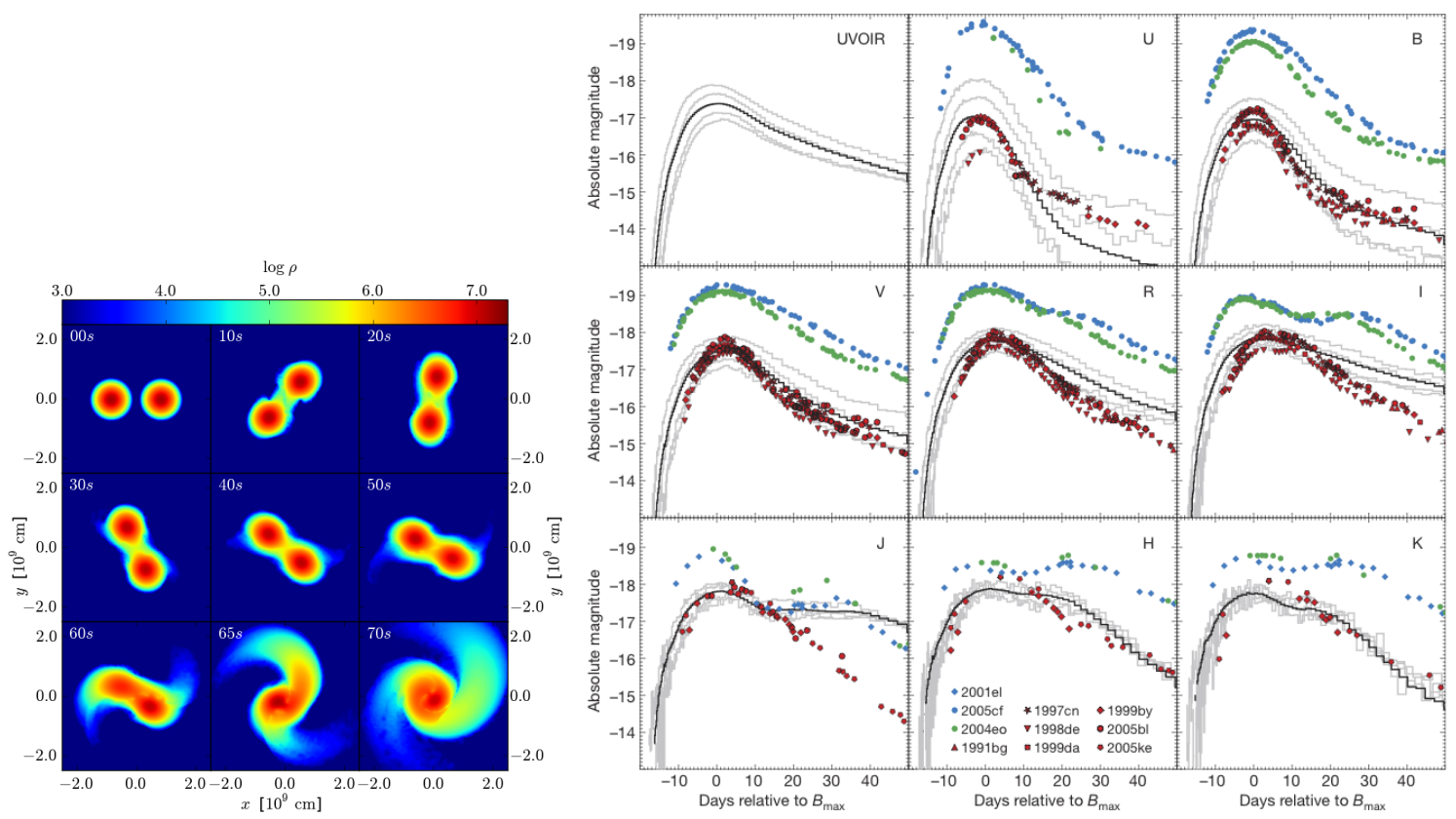

Figure 1: Merger of two $0.9 M_{\odot}$ WDs: inspiral and merger phase (left) and predicted light curves (right). For each band, the gray light curves correspond to different viewing angles and the black curve represents the angle-average (from [14, 3]).

merger of two carbon-oxygen white dwarfs [10, 11]. Although not all of these mergers may lead to thermonuclear explosions [12], the parameter space is rich and has not been fully explored. Here, we focus on the violent merger scenario for which the mass ratio of the merging WDs is close to unity. Due to the symmetry in the initial setup, break-up of the lighter star and subsequent formation of an accretion disk around the heavier companion is avoided. Instead, the stars merge violently within a few orbits. This evolution is followed in simulations based on a modified version of the Smoothed Particle Hydrodynamics code Gadget [13]. During the inspiral, the two WDs are heavily deformed due to tidal interaction and finally plunge into each other.

The inspiral and merger for two WDs of $0.9 M_{\odot}$ each is shown in Fig. 1. When the two masses first touch, temperatures above $2.8 \times 10^{9} \mathrm{~K}$ are reached in material of $\rho=3.8 \times 10^{6} \mathrm{~g} \mathrm{~cm}^{-3}$. These conditions can be sufficient to trigger a detonation [15]. Since the formation of a detonation wave cannot be resolved in our simulations, we assume a detonation triggers and follow its propagation over the merged object by means of our grid-based hydrodynamical supernova code LEAFS. The result is a powerful explosion of the merged object. Given the masses of the two initial WDs, the total mass of the ejecta is $1.8 M_{\odot}$ - exceeding the Chandrasekhar-mass. Nonetheless, the ensuing event is not a bright supernova. Since the density in the merged object is not very high, the amount of produced ${ }^{56} \mathrm{Ni}$ (powering the optical display by its radioactive decay) is only about $0.1 M_{\odot}$. Light curves derived from the explosion model with the radiative transfer code ARTIS [9] are shown in Fig. 1. They are much fainter than the light curves of normal SNe Ia but compare favorably to those of SN 1991bg-like objects. No theoretical model was previously available for this sub-class of SNe Ia, and so violent WD-WD mergers seem a promising option to explain them [3]. 


\section{Chandrasekhar-mass models}

Given the low rate expected for mergers of more massive WDs, different scenarios seem necessary to model normal SNe Ia. The long-time favorite and best explored option is that of Chandrasekhar-mass explosions where a carbon-oxygen WD accretes matter from a companion star (either $\mathrm{H}$ and $\mathrm{He}$ which then burn hydrostatically at the surface of the WD to $\mathrm{C}$ and $\mathrm{O}$, or directly $\mathrm{C}+\mathrm{O}$ material from another WD). When approaching the Chandrasekhar mass, the central density in the core increases dramatically. After a century of convective carbon burning (see the contribution of M. Zingale) a thermonuclear burning front is ignited by thermonuclear runaway in one or multiple sparks near the center of the WD.

\subsection{Pure deflagrations}

The nucleosynthesis in a prompt detonation of a Chandrasekhar-mass WD in hydrostatic equilibrium is inconsistent with observed spectra of SNe Ia. To synthesize appreciable amounts of intermediate-mass elements (such as $\mathrm{Si}, \mathrm{S}$, and $\mathrm{Ca}$ ), pre-expansion of the fuel material is required. This is possible if the burning starts out subsonically, as a deflagration flame. When propagating through the WD, the flame will be subject to buoyancy instabilities. In the non-linear regime, the Rayleigh-Taylor instability leads to the formation of mushroom-shaped bubbles of burning material that rise into the fuel. The shear flow ensuing at the interface of those bubbles (characterized by a Reynolds number of $\sim 10^{14}$ ) inevitably generates strong turbulent eddies that decay forming a turbulent energy cascade. In a wide sub-range of this cascade, the flame interacts with turbulent eddies of various sizes. They drag the flame around and lead to a drastic increase of flame surface area. As a consequence, the effective burning rate is strongly enhanced and the flame is accelerated beyond the laminar flame speed. This effect is crucial for a successful explosion of the WD.

An example of a simulation following a deflagration in a Chandrasekhar-mass WD is shown in Fig. 2. The propagation of the deflagration front through the WD is modeled with our grid-based hydrodynamic SN Ia code LEAFS. This simulation is prototypical for deflagration models that are ignited in multiple sparks around the center of the WD. The ${ }^{56} \mathrm{Ni}$ production amounts to $0.32 M_{\odot}$. Due to large-scale buoyancy instabilities, the chemical composition of the ejecta is strongly mixed. Thus from such simulations, only a faint, low energy, peculiar event can be expected. This is confirmed after nucleosynthesis postprocessing and mapping into our radiative transfer code ARTIS. Although the mapping procedure is non-trivial and still preliminary, the derived spectra (see right plot of Fig. 2) qualitatively resemble those observed for the SN Ia subclass of 2002cx-like events (of which SN 2005hk is used as an example here). This supports the idea that this subclass can be explained by deflagrations in WDs [16].

\subsection{Delayed detonations}

To reach the brightnesses and explosion energies of normal SNe Ia in Chandrasekhar-mass models, an enhanced burning is required. One possibility is the delayed detonation scenario $[17,18]$. After initial burning in the deflagration mode, the flame undergoes a transition to a supersonic detonation in a late stage of the explosion. The question of whether or not such deflagrationto-detonation transitions occur in SNe Ia is not answered yet. Recent studies [19, 20, 21], however, 

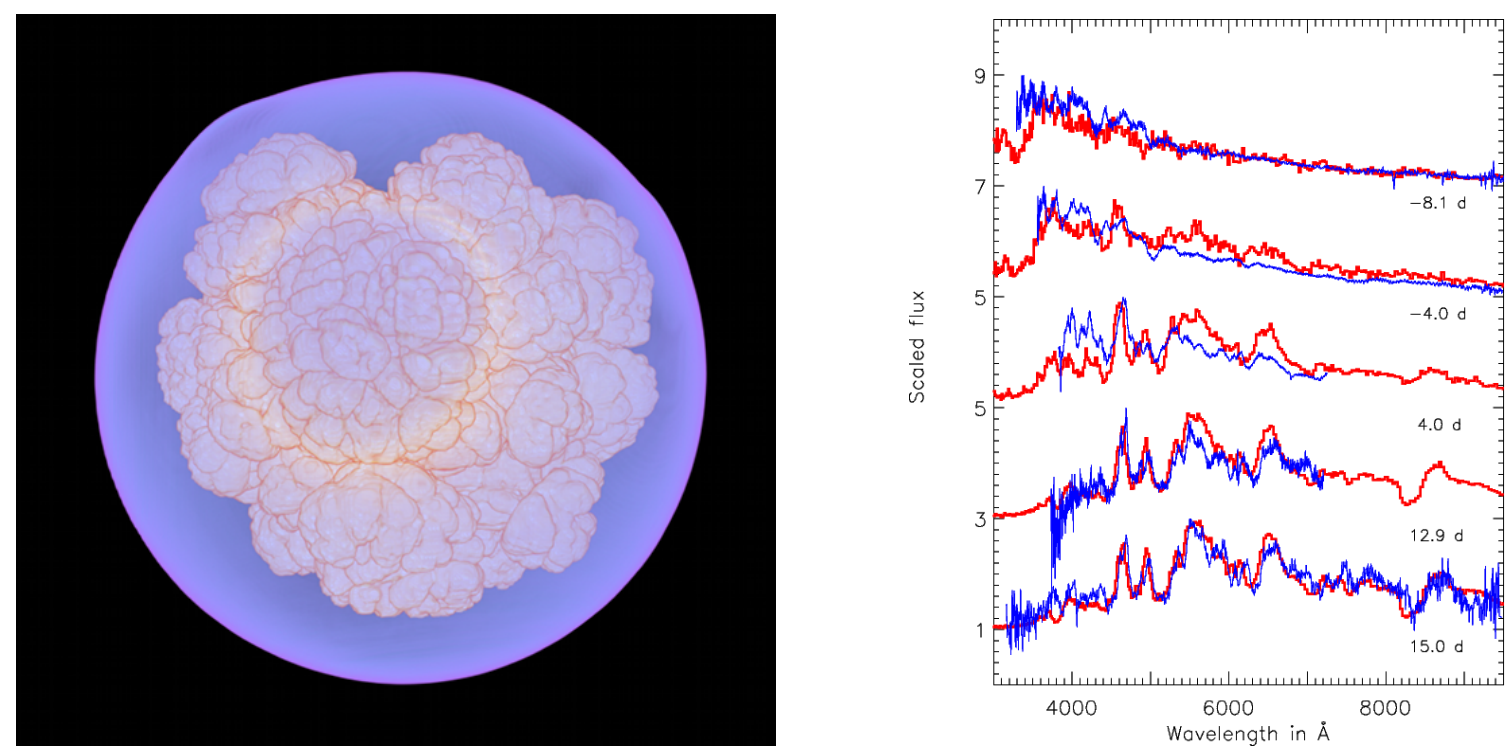

Figure 2: Pure deflagration in a Chandrasekhar-mass C+O WD: snapshot of the flame propagation $1.0 \mathrm{~s}$ after ignition in multiple sparks around the star's center (left) and preliminary predictions for spectra resulting from this event (right). The model spectra in red are compared to observations of SN 2005hk in blue. Note that this comparison does not reach the same quality as the others shown in this article. Plotted is only a scaled flux; the absolute magnitudes do not match. Moreover, no reddening correction was applied to the spectra of SN 2005hk (figure from [14]).

indicate that they may be possible. If so, the delayed detonation scenario holds promise for explaining the main characteristics of normal SNe Ia [22, 23, 24, 25, 26, 27]. Light curves and spectra predicted from these events [8] match the observations reasonably well and even qualitatively reproduce the width-luminosity relation [28] that is used to calibrate $\mathrm{SNe}$ Ia as distance indicators in cosmology. A potential problem for this scenario, however, is that some population synthesis studies predict too few realizations of progenitor systems able to form a Chandrasekhar-mass WD to be compatible with the observed rate of normal SNe Ia [2].

\section{Sub-Chandrasekhar mass models}

Sub-Chandrasekhar mass models were studied in the 1990s [29, 30] but have subsequently received little attention until recently [31]. From a population synthesis point of view, they seem attractive for explaining the bulk of SNe Ia [32]. The problem with this progenitor channel is that the nucleosynthesis expected from the detonation of a massive $\mathrm{He}$ shell above a $\mathrm{C}+\mathrm{O}$ core is inconsistent with the observations $[33,34,35]$. Previous models predicted a substantial ${ }^{56} \mathrm{Ni}$ production in this layer. Recently, models with larger core masses (around $1 M_{\odot}$ ) have been studied - these have the advantage that a thin He shell may trigger a detonation [36]. Although the He shell detonation then does not produce significant amounts of ${ }^{56} \mathrm{Ni}$, other iron-group isotopes are still synthesized [37]. In particular, absorption by $\mathrm{Cr}$ and $\mathrm{Ti}$ leads to strong flux redistribution towards the red parts of the spectrum making the models inconsistent with observations (see left plot of Fig. 3). One way to alleviate this problem is to start from a carbon-enriched He shell instead 

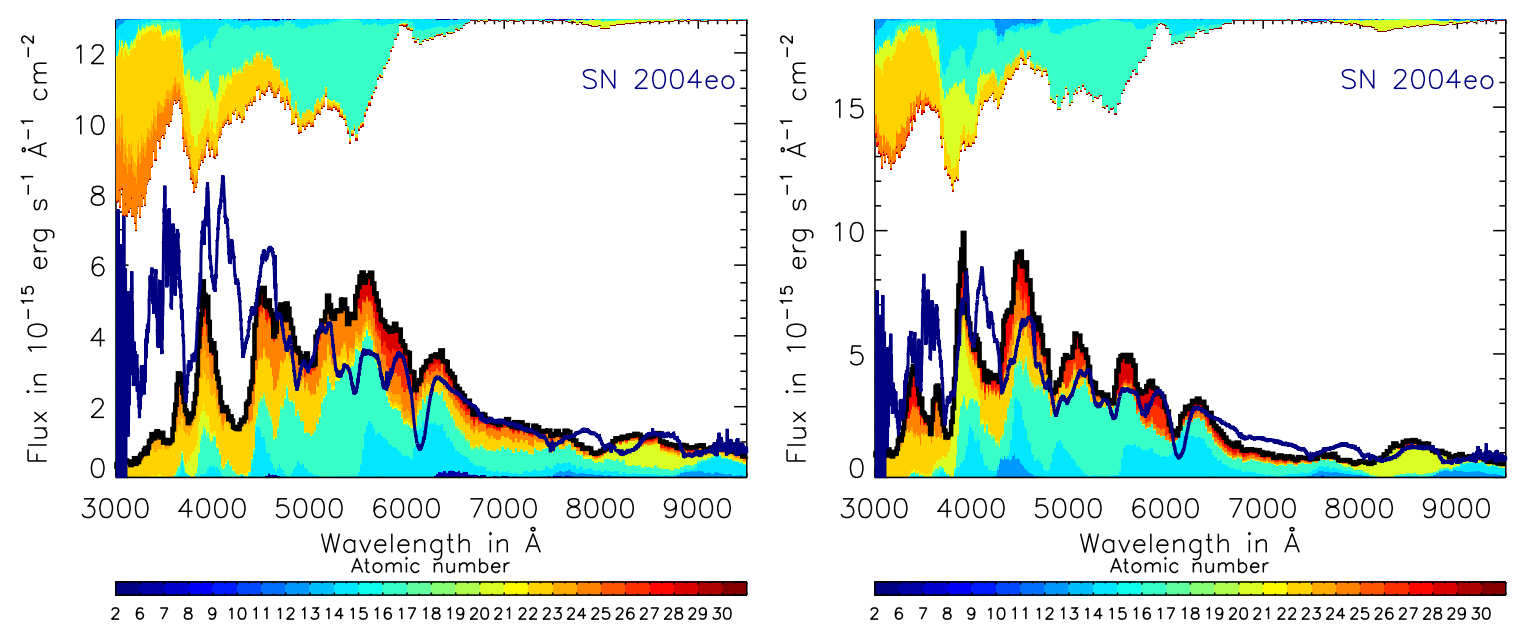

Figure 3: Synthetic spectra around $B$-band maximum from a sub- $\mathrm{M}_{\mathrm{Ch}}$ mass model $\left(M_{\text {core }}=1.025 M_{\odot}\right.$, $M_{\text {shell }}=0.055 M_{\odot}$ ) compared to a spectrum from SN 2004eo at 3 days before $B$-band maximum. Left: pure He shell, right: He shell with 33\% C admixture (figure from [14]).

of pure He [35]. This restricts the mass number of alpha elements that can be synthesized and with a $\sim 33 \%$ admixture of $\mathrm{C}$, the spectrum agrees much better with the observations (see right plot of Fig. 3). The capability of sub-Chandrasekhar mass models to reproduce characteristics (and variations) of the observed sample of normal SNe Ia can be demonstrated on the basis of detonations in bare $\mathrm{C}+\mathrm{O}$ cores [38].

\section{Conclusion}

A variety of progenitor systems and explosion mechanisms for SNe Ia have been proposed over the last few decades. One way of clarifying which of these contribute to the observed SN Ia sample is to follow the explosion, nucleosynthesis, and radiative transfer in a pipeline of simulations. This allows for a direct comparison of predictions with observations.

More detailed and comprehensive theoretical studies are still required but it seems that each the models discussed here finds a correspondence in the observed sub-classes of SNe Ia. A potential explanation is that the wealth of data from supernova surveys contains a wide range of possibilities that are realized in Nature. However, it could also be that currently available observational data are not sufficient to discriminate the models. In particular spectra around peak luminosity test mainly the outer layers of the ejecta and are not sensitive to their central regions where the explosion mechanism leaves complementary imprints. Therefore, the comparison between models and observations has to be extended. Nebular spectra and spectropolarimetry data seem promising for breaking the degeneracy of the models.

\section{Acknowledgments}

This work was supported by the Deutsche Forschungsgemeinschaft via the Emmy Noether Program (RO 3676/1-1), the Excellence Cluster EXC 153, and the Transregional Collaborative Research Center TRR 33. 


\section{References}

[1] W. Hillebrandt and J. C. Niemeyer. Type Ia Supernova Explosion Models. ARA\&A, 38 (2000) 191. [arXiv:astro-ph/0006305].

[2] A. J. Ruiter, K. Belczynski and C. Fryer. Rates and Delay Times of Type Ia Supernovae. ApJ, 699 (2009) 2026. [0904.3108].

[3] R. Pakmor, M. Kromer, F. K. Röpke, S. A. Sim, A. J. Ruiter et al. Sub-luminous type Ia supernovae from the mergers of equal-mass white dwarfs with mass $\sim 0.9 M_{\odot}$. Nature, 463 (2010) 61. [0911.0926].

[4] M. Reinecke, W. Hillebrandt and J. C. Niemeyer. Refined numerical models for multidimensional type Ia supernova simulations. A\&A, 386 (2002) 936. [arXiv : astro-ph/0111475] .

[5] C. Travaglio, W. Hillebrandt, M. Reinecke and F.-K. Thielemann. Nucleosynthesis in multi-dimensional SN Ia explosions. A\&A, 425 (2004) 1029. [arXiv: astro-ph/0 406281 ].

[6] F. K. Röpke, M. Gieseler, M. Reinecke, C. Travaglio and W. Hillebrandt. Type Ia supernova diversity in three-dimensional models. A\&A, 453 (2006) 203. [arXiv:astro-ph/ 0506107 ] .

[7] K. Maeda, F. K. Röpke, M. Fink, W. Hillebrandt, C. Travaglio et al. Nucleosynthesis in Two-Dimensional Delayed Detonation Models of Type Ia Supernova Explosions. ApJ, 712 (2010) 624. [1002.2153].

[8] D. Kasen, F. K. Röpke and S. E. Woosley. The diversity of type Ia supernovae from broken symmetries. Nature, 460 (2009) 869. [0907 . 0708 ] .

[9] M. Kromer and S. A. Sim. Time-dependent three-dimensional spectrum synthesis for Type Ia supernovae. MNRAS, 398 (2009) 1809. [0906.3152] .

[10] W. Benz, A. G. W. Cameron, W. H. Press and R. L. Bowers. Dynamic mass exchange in doubly degenerate binaries. I. 0.9 and 1.2 solar mass stars. ApJ, 348 (1990) 647.

[11] P. Lorén-Aguilar, J. Isern and E. García-Berro. High-resolution Smoothed Particle Hydrodynamics simulations of the colaescence of double white dwarfs. American Institute of Physics Conference Series, 1122 (2009) 320.

[12] H. Saio and K. Nomoto. Evolution of a merging pair of $C+O$ white dwarfs to form a single neutron star. A\&A, 150 (1985) L21.

[13] V. Springel. The cosmological simulation code GADGET-2. MNRAS, 364 (2005) 1105. [arXiv:astro-ph/0505010].

[14] F. K. Röpke, I. R. Seitenzahl, S. Benitez, M. Fink, R. Pakmor et al. Modeling Type Ia supernova explosions. Prog. Part. Nucl. Phys., (2011). In print, DOI 10.1016/j.ppnp.2011.01.026.

[15] I. R. Seitenzahl, C. A. Meakin, D. M. Townsley, D. Q. Lamb and J. W. Truran. Spontaneous Initiation of Detonations in White Dwarf Environments: Determination of Critical Sizes. ApJ, 696 (2009) 515. [0901.3677].

[16] M. M. Phillips, W. Li, J. A. Frieman, S. I. Blinnikov, D. DePoy et al. The Peculiar SN 2005hk: Do Some Type Ia Supernovae Explode as Deflagrations? PASP, 119 (2007) 360. [arXiv:astro-ph/0611295].

[17] S. I. Blinnikov and A. M. Khokhlov. Stage of spontaneous flame propagation in supernovae. Soviet Astronomy Letters, 13 (1987) 364. 
[18] A. M. Khokhlov. Delayed detonation model for type Ia supernovae. A\&A, 245 (1991) 114.

[19] F. K. Röpke. Flame-driven Deflagration-to-Detonation Transitions in Type Ia Supernovae? ApJ, 668 (2007) 1103. [arXiv:0709.4095].

[20] S. E. Woosley. Type Ia Supernovae: Burning and Detonation in the Distributed Regime. ApJ, 668 (2007) 1109. [arXiv:0709.4237].

[21] S. E. Woosley, A. R. Kerstein, V. Sankaran, A. J. Aspden and F. K. Röpke. Type Ia Supernovae: Calculations of Turbulent Flames Using the Linear Eddy Model. ApJ, 704 (2009) 255. [0811.3610].

[22] V. N. Gamezo, A. M. Khokhlov and E. S. Oran. Three-dimensional Delayed-Detonation Model of Type Ia Supernovae. ApJ, 623 (2005) 337. [arXiv:astro-ph/ 0409598 ] .

[23] F. K. Röpke and J. C. Niemeyer. Delayed detonations in full-star models of type Ia supernova explosions. A\&A, 464 (2007) 683. [arXiv:astro-ph/0703378].

[24] P. A. Mazzali, F. K. Röpke, S. Benetti and W. Hillebrandt. A Common Explosion Mechanism for Type Ia Supernovae. Science, 315 (2007) 825. [arXiv: astro-ph/ 0702351 ] .

[25] E. Bravo and D. García-Senz. A three-dimensional picture of the delayed-detonation model of type Ia supernovae. A\&A, 478 (2008) 843. [arXiv:0712.0510].

[26] K. Maeda, S. Benetti, M. Stritzinger, F. K. Röpke, G. Folatelli et al. An asymmetric explosion as the origin of spectral evolution diversity in type Ia supernovae. Nature, 466 (2010) 82. [1006. 5888 ] .

[27] A. P. Jackson, A. C. Calder, D. M. Townsley, D. A. Chamulak, E. F. Brown et al. Evaluating Systematic Dependencies of Type Ia Supernovae: The Influence of Deflagration to Detonation Density. ApJ, 720 (2010) 99. [1007.1138].

[28] M. M. Phillips, P. Lira, N. B. Suntzeff, R. A. Schommer, M. Hamuy et al. The Reddening-Free Decline Rate Versus Luminosity Relationship for Type Ia Supernovae. AJ, 118 (1999) 1766. [arXiv:astro-ph/9907052].

[29] S. E. Woosley and T. A. Weaver. Sub-Chandrasekhar mass models for Type Ia supernovae. ApJ, 423 (1994) 371.

[30] E. Livne and D. Arnett. Explosions of Sub-Chandrasekhar Mass White Dwarfs in Two Dimensions. ApJ, 452 (1995) 62.

[31] M. Fink, W. Hillebrandt and F. K. Röpke. Double-detonation supernovae of sub-Chandrasekhar mass white dwarfs. A\&A, 476 (2007) 1133. [arXiv:0710.5486].

[32] A. J. Ruiter, K. Belczynski, S. A. Sim, W. Hillebrandt, C. L. Fryer et al. Type Ia Supernova Delay Times and Rates for Sub-Chandrasekhar Mass Models (2010). Submitted to MNRAS, [1011.1407].

[33] P. Hoeflich, A. Khokhlov, J. C. Wheeler, M. M. Phillips, N. B. Suntzeff et al. Maximum Brightness and Postmaximum Decline of Light Curves of Type IA Supernovae: A Comparison of Theory and Observations. ApJ, 472 (1996) L81. [arXiv:astro-ph/9609070 ].

[34] A. M. Khokhlov, E. S. Oran and J. C. Wheeler. Deflagration-to-Detonation Transition in Thermonuclear Supernovae. ApJ, 478 (1997) 678. [arXiv: astro-ph/9612226].

[35] M. Kromer, S. A. Sim, M. Fink, F. K. Röpke, I. R. Seitenzahl et al. Double-detonation Sub-Chandrasekhar Supernovae: Synthetic Observables for Minimum Helium Shell Mass Models. ApJ, 719 (2010) 1067. [1006.4489]. 
[36] L. Bildsten, K. J. Shen, N. N. Weinberg and G. Nelemans. Faint Thermonuclear Supernovae from AM Canum Venaticorum Binaries. ApJ, 662 (2007) L95. [arXiv : astro-ph/0703578] .

[37] M. Fink, F. K. Röpke, W. Hillebrandt, I. R. Seitenzahl, S. A. Sim et al. Double-detonation sub-Chandrasekhar supernovae: can minimum helium shell masses detonate the core? A\&A, $\mathbf{5 1 4}$ (2010) A53.

[38] S. A. Sim, F. K. Röpke, W. Hillebrandt, M. Kromer, R. Pakmor et al. Detonations in Sub-Chandrasekhar-mass C+O White Dwarfs. ApJ, 714 (2010) L52. [1003.2917]. 